\title{
Metformin ameliorates diabetes but does not normalize the decreased GLUT 4 content in skeletal muscle of obese (fa/fa) Zucker rats
}

\author{
A.Handberg ${ }^{1}$, L.Kayser ${ }^{2}$, P.E.Høyer ${ }^{2}$, M. Voldstedlund ${ }^{1}$, H.P.Hansen ${ }^{1}$, J. Vinten ${ }^{1}$ \\ ${ }^{1}$ Institute of Medical Physiology B, Panum Institute, University of Copenhagen, Copenhagen, Denmark \\ ${ }^{2}$ Institute of Medical Anatomy A, Panum Institute, University of Copenhagen, Copenhagen, Denmark
}

\begin{abstract}
Summary. We studied the expression of the glucose transporter GLUT 4 in the soleus and red gastrocnemius muscles from obese, diabetic (fa/fa) Zucker rats compared to their lean littermates $(\mathrm{Fa} /-$ ), with and without treatment with the antidiabetic drug metformin. In the untreated groups of rats, the GLUT 4 content in a crude membrane fraction of both the soleus and the red gastrocnemius muscles were significantly lower in the obese (fa/fa) rats $(3.46 \pm 0.28$ vs. $6.04 \pm 0.41, p<0.001$ and $6.0 \pm 0.24$ vs. $9.1 \pm 0.48, p<0.0001$, respectively). Differences in GLUT 4 expression in soleus muscle from the same rats were confirmed by quantitative immunofluorescence microscopy, and the results were significantly correlated with the results obtained from quantitative immunoblotting (rho $=0.70, p<0.0005$ ). The decreased
\end{abstract}

expression of GLUT 4 in fa/fa rats could contribute to the well-established insulin resistance in skeletal muscle of these animals. After 4 weeks of treatment with metformin, weight gain was not affected in either the diabetic (fa/fa) rats or the lean $(\mathrm{Fa} /-)$ rats. Improvement of glucose homeostasis by metformin was not associated with normalization of the GLUT 4 expression in the skeletal muscles studied, indicating (1) that the decreased GLUT 4 expression is not directly related to hyperinsulinaemia and diabetes mellitus and (2) that metformin does not normalize the expression of GLUT 4 in skeletal muscle of the diabetic (fa/fa) Zucker rats.

Key words: GLUT 4, glucose transporter, insulin resistance, insulin action, Zucker rat, metformin, skeletal muscle.
Metformin (N,N-dimethylbiguanide) is an antidiabetic drug widely used to treat obese non-insulin-dependent diabetic patients. No stimulatory effect of metformin on insulin secretion has been demonstrated [1-4] and the antihyperglycaemic effect has been attributed to a variety of actions, including diminished intestinal absorption of carbohydrates [2,5] and reduced hepatic gluconeogenesis $[3,6-8]$. In vivo and in vitro studies have also indicated that metformin stimulates the insulin-induced glucose uptake in skeletal muscle and adipose tissue [2,9-12]. Insulin stimulation of glucose uptake in these tissues involves redistribution of the insulin responsive glucose transporter GLUT 4 from an intracellular pool to the plasma membrane as reviewed by Burant et al. [13]. However, studies on different types of muscles from rats have shown that insulin responsiveness is also related to the content of transporter protein in crude membranes $[14,15]$.

The obese Zucker rat (fa/fa) is a well-characterized animal model of insulin resistance in skeletal muscle, and the adult Zucker rats display severe hyperinsulinaemia, glucose intolerance and an elevated plasma insulin response to a glucose challenge [16-18]. It has been suggested that the insulin resistance is partly the result of an impairment of insulin stimulation of the glucose transport [19-21]. We studied the expression of GLUT 4 in skeletal muscles from obese Zucker rats and their lean littermates, with and without treatment with metformin, to establish whether the impaired insulin action in skeletal muscle is associated with a decreased glucose transporter content, and to investigate the effect of the antidiabetic drug metformin on the GLUT 4 content in this tissue. In addition to conventional Western blotting of a crude muscle membrane fraction, the GLUT 4 content in cryosections of muscle was determined by use of a new, in this context, approach: quantitative immunofluorescence microscopy.

\section{Materials and methods}

Housing, care and medication of animals. Obese male Zucker rats (fa/fa) and theirlean littermates ( $\mathrm{Fa} /$-) were purchased from Charles River Laboratories (Cambridge, Mass., USA) at 8 weeks of age. They were housed two three per cage and were provided with free access to food (crude protein $23 \%$, crude fat $5 \%$ and carbohydrate $47.5 \%$ by weight, the remaining being moisture and fibres) and water. The temperature of the animal room was kept at $21^{\circ} \mathrm{C}$, with an artificial 12:12-h light-dark cycle. Seven obese Zucker rats and seven 
lean control rats were randomly chosen for medication, and the remaining seven rats from each group served as controls. The rats assigned for medical treatment were given metformin dissolved in $0.9 \% \mathrm{NaCl}$ every morning during a period of 24 days while the controls were given vehicle. Metformin was administered by use of a gastric tube, in doses starting with $90 \mathrm{mg} /$ day for 6 days increasing to $180 \mathrm{mg} /$ day for 6 days, $225 \mathrm{mg} /$ day for 6 days and finally $270 \mathrm{mg} /$ day for the remainder of the study period. The last dose of metformin or vehicle was given $24 \mathrm{~h}$ before the day of study. The animals were weighed twice weekly.

Tissue and blood sampling. After an overnight fast, the animals were anaesthetized by pentobarbital sodium $(50 \mathrm{mg} / \mathrm{kg}$ body weight i.p.) and weighed. Blood samples for determination of insulin were collected in iced tubes containing 500 kallikrein inhibitor units (KIU) Trasylol (Bayer, Leverkusen, FRG) and $1.5 \mathrm{mg}$ EDTA per $\mathrm{ml}$ of blood, while samples for determination of glucose and fructosamine were stabilized with $10 \mathrm{IU}$ heparin per ml of blood. The soleus and red gastrocnemius muscles were excised and immediately frozen by immersion in $n$-hexane at $-70^{\circ} \mathrm{C}$ and stored at the same temperature until analysis. Following excision of muscles, the animals were killed by excision of the heart.

Analysis of blood samples. A commercially available RIA kit was used for determination of insulin as described by the manufacturer (Novo-Nordisk, Bagsvaerd, Denmark) using rat insulin as standard. Glucose was measured by an automated glucose analyser (YSI 23AM; Yellow Springs Instruments, Yellow Springs, Ohio, USA), and glycated fructosamine was analysed using a commercially available kit (Precimat; Boehringer Mannheim, Meylan, France), and data were normalized to the concentration of plasma protein.

Immunoblotting. Frozen soleus of red gastrocnemius muscles were homogenized for $7 \mathrm{~s}$ using a Polytron (n. Prof. P. Willems, Luzern, Switzerland) (max. setting) in $3 \mathrm{ml}$ of $50 \mathrm{mmol} / \mathrm{l}$ Hepes, $\mathrm{pH} 7.6$, $250 \mathrm{mmol} / 1$ sucrose, $10 \mathrm{mmol} / 1 \mathrm{EDTA}, 1.5 \mathrm{mmol} / \mathrm{l}$ phenylmethylsulphonyl-fluoride (PMSF) and $400 \mathrm{KIU} / \mathrm{ml}$ Trasylol. A crude membrane fraction, consisting of a mixture of plasma membrane and intracellular membrane, was isolated from the homogenate by a centrifugation procedure, essentially as described by Klip et al. [22] with previously published modifications $[23,24]$. The homogenates were centrifuged at $1,000 \mathrm{~g}$, for $10 \mathrm{~min}$, at $4^{\circ} \mathrm{C}$, followed by a centrifugation of the resulting supernatants for $10 \mathrm{~min}, 9,000 \mathrm{~g}$, at $4^{\circ} \mathrm{C}$. The supernatants underwent a final spin at $200,000 \mathrm{~g}$, for $2 \mathrm{~h}$, at $4^{\circ} \mathrm{C}$ and the resulting pellets were resuspended in homogenization buffer without protease inhibitors. Protein content was determined by the Coomassie brilliant blue G-250 dye method using the kit from Biorad (Richmond, Calif., USA). Protease inhibitors were added to the remaining crude membrane preparation $(1.5 \mathrm{mmol} / \mathrm{PMSF}$ and $400 \mathrm{KIU} / \mathrm{ml}$ Trasylol). Samples containing $50 \mu \mathrm{g}$ of protein from each crude membrane preparation were analysed in duplicate by sodium-dodecyl-sulphate polyacrylamide gel electrophoresis (SDSPAGE) and Western blotting $[23,24]$. The antibody used for detection of the glucose transporter GLUT 4 was an affinity-purified polyclonal rabbit antibody directed against an artificial peptide, $\mathrm{P} 1$, identical to the $13 \mathrm{COOH}$-terminal amino acids of GLUT 4 [23]. Quantitation of immunoreactivity was performed by densitometric scanning using a preparation of adipocyte plasma membrane as internal standard. No significant differences in protein recovery in the crude membrane fractions between the four groups of rats were found in either soleus or red gastrocnemius muscle (data not shown).

Fluorescence labelling of GLUT 4 in tissue sections. Soleus muscles were divided into three tissue blocks by two transverse cuts at random positions on the long axis of the muscle. From each tissue block, six cryosections were cut $6 \mu \mathrm{m}$ thick and all sections from one soleus muscle were adsorbed on the same gelatin-coated slide. Slides were incubated with Tris-buffered saline (TBS; $10 \mathrm{mmol} / \mathrm{I}$ Tris- $\mathrm{HCl}$, pH 7.4 and $0.9 \% \mathrm{NaCl}$ ) containing $5 \%$ bovine serum albumin (BSA) for $30 \mathrm{~min}$ at room temperature to block non-specific binding, essentially as previously described [24]. Quadruplicate sections from each tissue block (in total 12 sections on each slide) were incu- bated for $1 \mathrm{~h}$ with the above-described polyclonal antibody towards the COOH-terminal part of GLUT 4, diluted $1: 100$ in TBS with $1 \%$ BSA. Duplicate sections from each tissue block (in total six sections on each slide) were incubated with dilution buffer alone and served as background sections. The cryosections were washed three times for $3 \mathrm{~min}$ with TBS followed by incubation with a fluorescence conjugated goat anti-rabbit IgG antibody (BODIPY [B2752]; Molecular Probes, Eugene, Ore., USA) for $1 \mathrm{~h}$. Finally, the sections were rinsed in TBS and mounted in Aquamount (BDH, Poole, Dorset, UK). The sections were kept in the dark at $-20^{\circ} \mathrm{C}$ until analysis by fluorescence microscopy.

Quantitation of immunofluorescence labelling in tissue sections Instrumentation. The fluorescence intensity emitted from the specifically bound secondary antibody was measured using a Zeiss Axiovert 10 epifluorescense microscope equipped with a Plan Neofluar water immersion objective (Magnification $\times 25$, numerical aperture 0.80 ), a XBO $75 \mathrm{~W}$ light source, a fast-shutter and a Dage/MTI SIT 66 camera (Michigan City, Ind., USA) connected to a MVP-AT version 10 digitizer (Matrox Electronic Systems Limited, Dorval, Quebec, Canada). The following set of filters was used: excitation $485 / 20 \mathrm{~nm}$, beamsplitter $510 \mathrm{~nm}$ and emission $515-565 \mathrm{~nm}$ (Zeiss, Oberkochen, FRG). The system was operated by the software package IMAGE1/AT 4.01b (Universal Imaging Corporation, West Chester, Pa., USA).

Quantitation of fluorescence. The background signal from the videocamera was adjusted to a black level of zero. using back-ground sections (see above). The high voltage (668 keV) and gain (4.86) were adjusted such that the main part of the measurements were within the midrange of the camera sensitivity [25]. From each slide, in $3 \times 3$ of the specifically-labelled sections on a slide, an area was randomly selected and an image was acquired by averaging and digitizing 16 videoframes (PAL; 25 frames/second). The total illumination time was $1.5 \mathrm{~s}$. The resulting image $(512 \times 512$ pixels $)$ was stored on a hard disk. After acquisition of the images, a dark and a white reference image was obtained using an uranylglass without and with illumination, respectively, to be used for background and shading corrections [25]. After the image acquisition, the intensity of fluorescence was quantitated using shading and background corrected images. The immunofluorescence intensity was discriminated using a level between 100 and 255 grey values. This signal does not represent the total amount of fluorescent secondary antibody but gives a measure of total GLUT 4, and the level is suitable to detect differences of GLUT 4 between the groups, effectively eliminating background signals. In each image the intensity in two thirds of the image area was measured and integrated. The sum of the integrated intensities from nine images was taken to represent a muscle. Sections from one rat from the untreated $\mathrm{Fa}$-control group and three rats from the treated control group were excluded a posteriori due to artifacts in the sections. Partial thawing of the sections during mounting on slides could cause intense and diffuse non-specific binding of the secondary fluorescent antibody.

\section{Statistical analysis}

Data in text, tables and figures are given as mean \pm SEM. Statistical significance between groups was tested by use of a Mann-Whitney test for unpaired comparisons, and Spearmans tho for correlation was used, One or two-sided tests were used where appropriate (indicated in text). Statistical analysis was performed using MEDSTAT version 2.1, programmed by H.R. Wulff and P.Schlichting, Herlev University Hospital (Herlev, Denmark).

\section{Results}

The diabetic (fa/fa) Zucker rats were significantly more obese than their lean littermates at the onset of metformin treatment (body weight (g) $367 \pm 16$ vs $277 \pm 6.5$, Table 1 ), 
Table 1. Characteristics of animals

\begin{tabular}{|c|c|c|c|c|}
\hline \multirow[b]{3}{*}{$n$} & \multicolumn{2}{|c|}{ Control $(\mathrm{Fa} /-)$ rats } & \multicolumn{2}{|c|}{ Obese (fa/fa) rats } \\
\hline & $\overline{\text { Metfor }}$ & $\min { }^{+}$ & \multicolumn{2}{|c|}{ Metformin } \\
\hline & 7 & 6 & 7 & 5 \\
\hline $\begin{array}{l}\text { Weight (g) } \\
\text { Start } \\
\text { End }\end{array}$ & $\begin{array}{l}278 \pm 10^{\mathrm{a}} \\
342 \pm 11^{\mathrm{a}}\end{array}$ & $\begin{array}{l}275 \pm 10 \\
329 \pm 12\end{array}$ & $\begin{array}{l}348 \pm 18 \\
482 \pm 10\end{array}$ & $\begin{array}{l}394 \pm 28 \\
523 \pm 22\end{array}$ \\
\hline $\begin{array}{l}\text { Fasting plasma } \\
\text { glucose (mmol/ })\end{array}$ & $6.4 \pm 0.3^{b}$ & $6.6 \pm 0.3$ & $12.7 \pm 1.0^{\mathrm{c}}$ & $8.9 \pm 1.0$ \\
\hline $\begin{array}{l}\text { Fasting plasma } \\
\text { insulin (ng/ml) }\end{array}$ & $2.8 \pm 0.6^{c}$ & $1.6 \pm 0.5$ & $10.9 \pm 2.7^{\circ}$ & $5.9 \pm 0.7$ \\
\hline Fructosamine & $155 \pm 8.4$ & $163.3 \pm 6.1$ & $186.8 \pm 12.6$ & $127.6 \pm 10.2$ \\
\hline
\end{tabular}

Data are shown as mean \pm SEM. See Materials and methods for metformin dosage and route of administration.

${ }^{a} p<0.00005$ vs untreated fa/fa rats, ${ }^{b} p<0.002$ vs untreated fa/fa rats, ${ }^{c} p<0.05$ vs fa/fa rats treated with metformin (one-sided Mann-Whitney), ${ }^{\mathrm{d}} p<0.01$ vs untreated fa/fa rats, ${ }^{\mathrm{e}} p<0.005$ vs metformin treated fa/fa rats (one-sided Mann-Whitney)

i.e. when the rats were approximately 8 weeks of age. The $\mathrm{fa} /$ fa rats gained relatively more weight during the study compared to the lean controls, and by the end of the study the weight of the fa/fa rats was $149 \%$ of control rats (Table 1). Metformin treatment did not influence weight gain during the study in the fa/fa Zucker rats or in the lean. controls (Table 1). Weight was positively correlated to fasting insulin at the end of the study (rho $=0.72$, $p<0.00005$ ). Fasting glucose levels were significantly elevated in the untreated fa/fa rats compared with control rats and glycated fructosamine tended to be increased in the diabetic rats (Table 1 ). Glycated fructosamine was significantly reduced by $30 \%$ by metformin treatment in the fa/fa rats (Table 1). Fasting blood glucose levels were lowered by metformin in the diabetic fa/fa rats $(8.9 \pm 1.0 \mathrm{vs}$ $12.7 \pm 1.0 \mathrm{mmol} / \mathrm{l})$, whereas no effect was found in the lean, non-diabetic rats. Metformin treatment also lowered fasting plasma insulin in the obese fa/fa rats $(10.9 \pm 2.7 \mathrm{vs}$ $5.9 \pm 0.7 \mathrm{ng} / \mathrm{ml}$ ), and was without effect in $\mathrm{Fa} /$ - rats (Table 1).

In untreated fa/fa rats, the content of GLUT 4 in a crude membrane fraction isolated from the soleus muscle was decreased to $57 \%$ of control $(3.46 \pm 0.28$ vs $6.04 \pm 0.41$, Fig. 1). Treatment with metformin did not significantly influence GLUT 4 expression in soleus muscle in either the diabetic fa/fa rats or in the controls $(\mathrm{Fa} /-)$. Similarly, in red gastrocnemius muscle, the GLUT 4 content in crude membranes was significantly lower in the untreated fa/fa rats compared with controls $(6.0 \pm 0.24$ vs $9.1 \pm 0.48$, Fig.1). Metformin caused no change in GLUT 4 expression in red gastrocnemius muscle in the diabetic group and in the lean controls even a reduction of $19 \%$ was demonstrated. The GLUT 4 content in untreated animals, when expressed relative to protein content in the crude membrane preparation, was significantly higher in red gastrocnemius compared to soleus muscle $(9.09 \pm 0.48$ vs $6.04 \pm 0.41, p<0.002$ in the $\mathrm{Fa} /-$ rats and $6.0 \pm 0.24$ vs $3.46 \pm 0.28, p<0.001$ in the fa/fa rats). In all rats taken together, GLUT 4 content was negatively

correlated to fasting plasma insulin in both the soleus (rho $=-0.5, p<0.02$ ) and the red gastrocnemius muscles (rho $=-0.56, p<0.005$ ) as well as to fasting plasma glucose in the soleus (rho $=-0.47, p<0.02$ ) and the red gastrocnemius muscles ( rho $=-0.58, p<0.005$ ).

Furthermore, the expression of GLUT 4 in the soleus muscle was also measured by quantitative immunofluorescence microscopy. The expression of GLUT 4, as measured in sections, was lower in muscle sections from the fa/fa obese rats compared with controls $(5.47 \pm 0.57 \mathrm{vs}$ $8.64 \pm 1.51, p<0.05$, one-sided Mann-Whitney). The content of GLUT 4 in soleus muscle determined in a crude membrane fraction by Western blotting correlated significantly with the content measured in the same muscles by

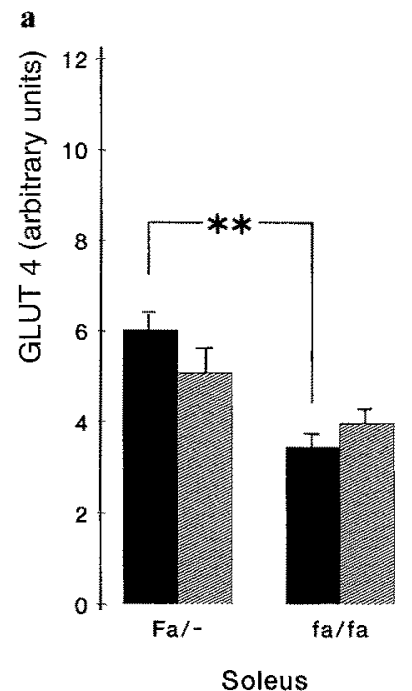

b

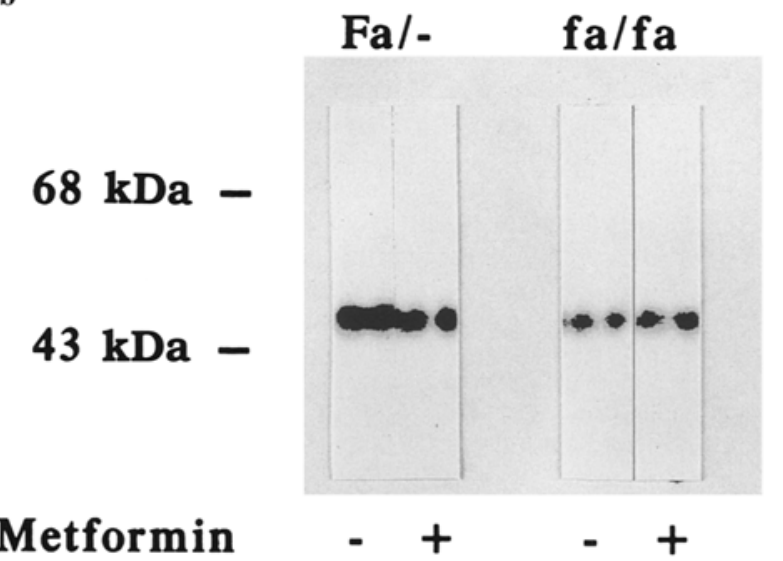

Fig.1a,b. Expression of GLUT 4 in soleus and red gastrocnemius muscle from obese (fa/fa) Zucker rats and their lean littermates. a GLUT 4 content in a crude membrane fraction from soleus (left panel) or red gastrocnemius (right panel) muscles was determined by quantitative immunoblotting, and the results, expressed relatively to protein content and to an internal standard, are given in arbitrary units at the vertical axis. fa/fa designates obese diabetic Zucker rats, and $\mathrm{Fa}$ /- their lean littermates, $\mathbf{n}$ the untreated groups and $\mathrm{O}$ the rats treated with metformin. Results are given as mean $\pm S E M$, $* p<0.05, * * p<0.001, * * * p<0.0001$. b Representative immunoblot of GLUT 4 in a crude membrane fraction of soleus muscle from obese diabetic Zucker rats ( $\mathrm{fa} / \mathrm{fa}$ ) and their lean littermates ( $\mathrm{Fa} /$-), with $(+)$ and without $(-)$ treatment with metformin (bottom). Determinations were performed in duplicate. Molecular weight markers are indicated on the left 

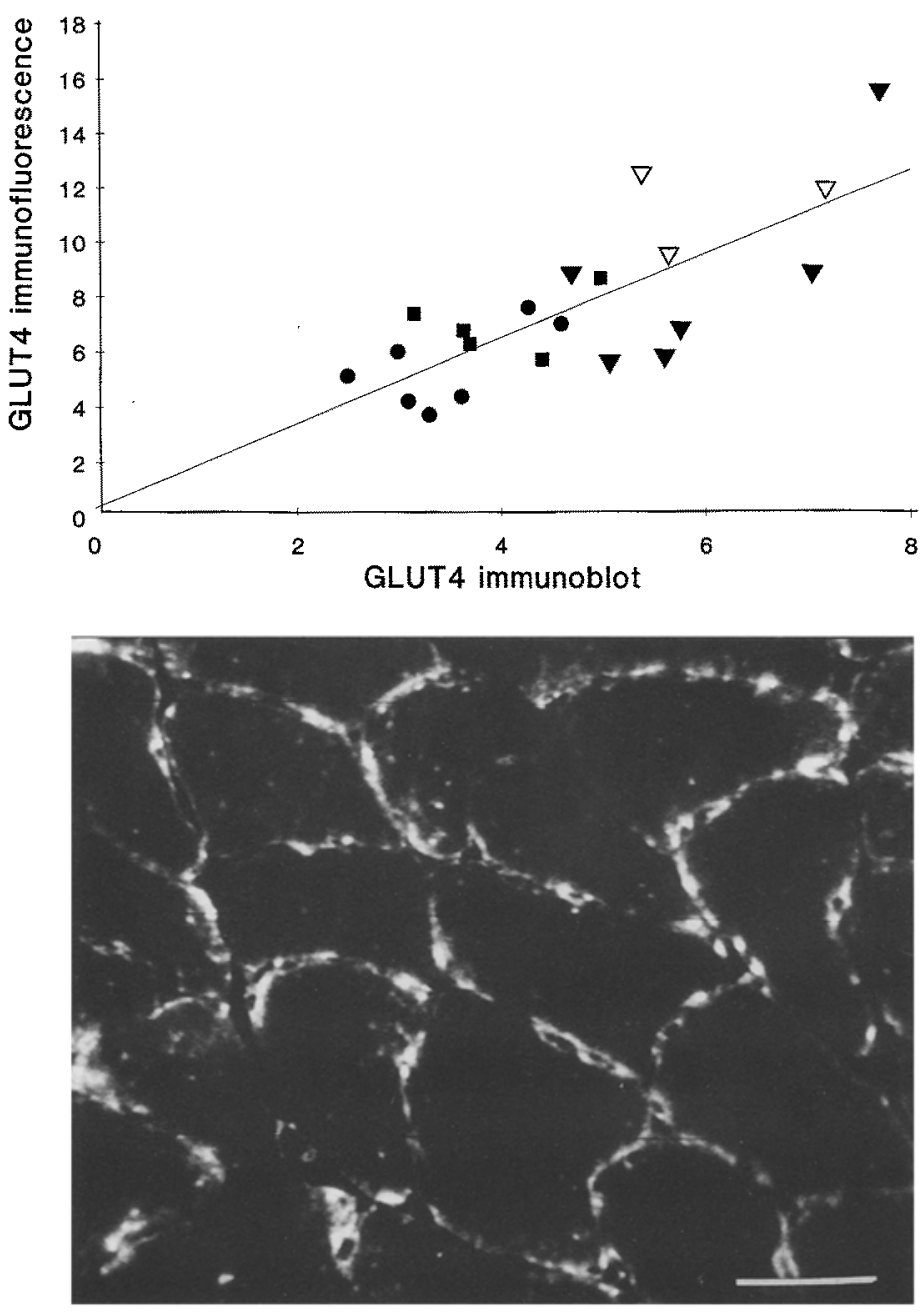

Fig. 2. Relationship between GLUT 4 content in the soleus muscles determined by quantitative immunoblotting and by quantitative immunofluorescence microscopy. Upper panel: The content of GLUT 4 in soleus muscles was determined in a crude membrane fraction by quantitative immunoblotting ( $x$-axis) and in sections of the contralateral muscle by quantitative immunofluorescence microscopy ( $y$-axis), and the results from the individual animals, expressed in arbitrary units, were significantly correlated (rho $=0.7$, $p<0.0005, d f 19)$. $\nabla, \mathrm{Fa} /$ - untreated rats; $\nabla \mathrm{Fa} /$, metformintreated rats; - fa/fa untreated rats; $\mathbf{w}$, fa/fa metformin-treated rats. Lower panel: Representative immunofluorescence labelling of GLUT 4 in a cryosection of soleus muscle from a Fa/-rat. Bar corresponds to $50 \mu \mathrm{m}$

quantitative immunofluorescence microscopy (rho $=0.7$, Fig.2).

\section{Discussion}

Insulin resistance is a major metabolic characteristic of non-insulin-dependent diabetes and obesity, and involves decreased sensitivity and/or responsiveness of the glucose transport system to insulin in target tissues [26, 27]. Skeletal muscle is the primary tissue responsible for insulinstimulated glucose disposal [28], where the glucose transporter GLUT 4 is believed to be responsible for insulin-stimulated glucose uptake [13]. Although translocation of GLUT 4 to the plasma membrane is believed to be an important step in the stimulation and regulation of glucose uptake by insulin, most studies of skeletal muscle have measured the level of GLUT 4 in crude membrane fractions rather than in plasma membranes, because the subcellular fractionation of skeletal muscle necessary for the study of translocation is technically difficult $[22,29$ 31]. However, studies on different types of muscles from rats have shown that insulin responsiveness is related to the level of transporter protein $[14,15]$.

Glucose transporter expression in skeletal muscle has been studied in man and in several animal models of insulin resistance. In non-insulin-dependent diabetes most studies agree that expression of GLUT 4 protein in skeletal muscle is normal $[23,32]$. In skeletal muscle from streptozotocin (STZ)-diabetic rats, however, previous reports have established a reduced expression of GLUT 4 protein and mRNA which are restored to normal levels with insulin therapy $[33,34]$.

In the present study, we have used the obese Zucker rat, which in the adult state is characterized by moderate hyperglycaemia and severe hyperinsulinaemia [16-18], as a model of insulin resistance. Using similar methods for quantitation of muscle GLUT 4 as in previous studies of Zucker rats $[35,36]$, we demonstrated a reduced expression of GLUT 4 in soleus and red gastrocnemius muscles. The validity of the present finding was confirmed by quantitative immunofluorescence microscopy. The individual measurements of GLUT 4 in soleus muscles obtained by the two methods were significantly correlated, and the decreased GLUT 4 expression was reproduced. Our result is in contrast to that of Friedman et al. [35], where GLUT 4 content in the gastrocnemius muscle of obese Zucker rats was normal compared to their lean littermates. This discrepancy could be due to a transiently decreased GLUT 4 expression as rats of considerably different age were studied ( 36 weeks vs 11-12 weeks in our study). A study of muscle insulin resistance and GLUT 4 expression in muscle from obese Zucker rats of increasing age (2170 days), however, demonstrated no difference in GLUT 4 content in the extensor digitorum longus muscle nor in the diaphragma at any timepoint [36].

Most studies of other hyperinsulinaemic animal models of insulin resistance have not suggested a decreased expression of GLUT 4 in skeletal muscle. Thus, in the obese $(\mathrm{db} / \mathrm{db})$ mouse [37] and in the viable yellow mouse [38], skeletal muscle GLUT 4 content was normal, and in rats made insulin resistant by long-term infusion of glucose [39], or by dexamethasone treatment [40] no effect on transporter expression was demonstrated. However, in insulin resistance induced by 4 days infusion of insulin in healthy rats, GLUT 4 protein was decreased in the tibialis muscle [41]. Additionally, in a new rat model of insulin resistance, the obese hypoinsulinaemic diabetic Zucker rat (ZDF/Drt-fa), GLUT 4 content was found to be reduced to about the same extent as in the present study [38,42]. Thus, the relation between insulin resistance and GLUT 4 expression differs between the models studied and may be related to the known variables of glycaemia, insulinaemia, corticosteronaemia etc. In view of the variety of animal 
models of insulin resistance previously studied under different conditions, and the conflicting conclusions concerning the relationship between GLUT 4 expression and insulin sensitivity drawn from these studies, the fact that the GLUT 4 expression reported here was demonstrated by use of two different methods, increases the reliability of the demonstrated decreased expression of GLUT 4 in skeletal muscle from fasting fa/fa Zucker rats.

It has been proposed that hyperinsulinaemia could contribute to the development and sustaining of muscle insulin resistance in genetically obese rats [36]. In support of this we demonstrated a negative correlation between GLUT 4 and fasting insulin and glucose. However, despite amelioration of diabetes and hyperinsulinaemia after 3 weeks of metformin treatment, normalization of GLUT 4 content in the fa/fa rats could not be demonstrated. This could be due to the fact that insulin levels were not fully normalized. The site of action of metformin has been subject to some discussion. Studies using the hyperinsulinaemic clamp technique have indicated that in non-insulin-dependent diabetes metformin augments the insulin-stimulated glucose uptake in muscle $[9,10]$. This finding is confirmed by incubations of muscle strips from non-diabetic subjects [12]. In rat adipocytes, short-term administration of metformin increases the insulin-stimulated glucose uptake by potentiating the insulin-stimulated translocation of transporters to the plasma membrane [11]. Our finding of lack of alteration in GLUT 4 content indicates that the level of action of metformin in skeletal muscle is not the expression of GLUT 4. However, as in adipocytes [11] metformin could potentiate insulin-stimulated translocation of GLUT 4.

In conclusion, we have demonstrated that the insulin resistance in skeletal muscle of the obese diabetic fa/fa Zucker rat, previously demonstrated by others, is associated with a decreased expression of GLUT 4 when compared to lean littermates. Amelioration of the hyperinsulinaemia and the diabetes by metformin treatment was not associated with normalization of the GLUT 4 expression in the skeletal muscles studied.

Acknowledgements. We thank J. Harpøth and $\mathrm{K}$. Clante for excellent technical assistance. The Novo-Nordisk Foundation and the Danish Diabetes Association are gratefully thanked for financial support of this study. The Velux Foundation of 1981 have donated the equipment for fluorescence microscopy. Dr. A. Handberg is a recipient of a research fellowship donated by the Novo Foundation.

\section{References}

1. Sterne J (1969) Pharmacology and mode of action of the hypoglycemic guanidine derivates. In: Campbell GD (ed) Oral hypoglycemic agents. Academic Press, New York, pp 193-245

2. Bailey CJ (1988) Metformin revisited: its actions and indications for use. Diabetic Med 5:315-320

3. Jackson RA, Hawa MI, Jaspan JB et al. (1987) Mechanism of metformin action in non-insulin-dependent diabetes. Diabetes 36: 632-640

4. Wu MS, Johnston P, Sheu WH-H et al. (1990) Effect of metformin on carbohydrate and lipoprotein metabolism in NIDDM patients. Diabetes Care 13:1-8
5. Lorch E (1971) Inhibition of intestinal absorption and improvement of oral glucose tolerance by biguanides in the normal and in the streptozotocin-diabetic rat. Diabetologia 7:195-203

6. Meyer F, Ipaktchi M, Caluser H (1967) Specific inhibition of gluconeogenesis by biguanides. Nature (Lond) 213: 203-204

7. DeFronzo RA, Barzilai N, Simonson DC (1991) Mechanism of metformin action in obese and lean noninsulin-dependent diabetic subjects. J Clin Endocrinol Metab 73: 1294-1301

8. Klip A, Leiter LA (1990) Cellular mechanism of action of metformin. Diabetes Care 13:696-704

9. Hother-Nielsen O, Schmitz O, Andersen PH, Beck-Nielsen $\mathrm{H}$, Pedersen O (1989) Metformin improves peripheral but not hepatic insulin action in obese patients with type II diabetes. Acta Endocrinol 120: 257-265

10. Pedersen O, Nielsen OH, Bak J, Richelsen B, Beck-Nielsen H, Schwartz-Sørensen N (1989) The effect of metformin on adipocyte insulin action and metabolic control in obese subjects with type II diabetes. Diabetic Med 6: 249-256

11. Matthaei S, Hamann A, Klein HH et al. (1991) Association of metformin's effect to increase insulin-stimulated glucose trans port with potentiation of insulin-induced translocation of glucose transporters from intracellular pool to plasma membrane in rat adipocytes. Diabetes 40:850-857

12. Galuska D, Zierath J, Thorne A, Sonnenfield T, Wallberg-Henriksson H (1991) Metformin increases insulin-stimulated glucose transport in insulin-resistant human skeletal muscle. Diabet Metab 17: 159-163

13. Burant CF, Sivitz WI, Fukumoto $H$ et al. (1991) Mammalian glucose transporters: structure and molecular regulation, Rec Prog Horm Res 47:349-387

14. Kern M, Wells JA, Stephens JM et al. (1990) Insulin responsiveness in skeletal muscle is determined by glucose transporter (GLUT 4) protein level. Biochem J 270: 397-400

15. Ploug T, Stallknecht BM, Pedersen O et al. (1990) Effect of endurance training on glucose transport capacity and glucose transporter expression in rat skeletal muscle. Am J Physiol 259: E778E786

16. Zucker LM, Antoniades HN (1972) Insulin and obesity in the Zucker genetically obese rat "fatty". Endocrinology 90: 1320 1330

17. Becker-Zimmerman K, Berger M, Berchtold P, Gries FA, Herberg L, Schwenen M (1982) Treadmill training improves intravenous glucose tolerance and insulin sensitivity in fatty Zucker rats. Diabetologia 22: 468-474

18. Ionescu E, Sauter JF, Jeanrenaud B (1985) Abnormal oral glucose tolerance in genetically obese (fa/fa) rats. Am J Physiol 248: E500-E506

19. Crettaz M, Prentki M, Zanietti D, Jeanrenaud B (1980) Insulin resistance in soleus muscles from obese Zucker rats. Involvement of several defective sites. Biochem $J 186: 525-534$

20. Penicaud L, Ferre P, Terretaz J et al. (1987) Development of obesity in Zucker rats: early insulin resistance in muscles but normal sensitivity in white adipose tissue. Diabetes 36: 626-631

21. Sherman WM, Katz AL, Cutler CL, Withers RT, Ivy JL (1988) Glucose transport: locus of muscle insulin resistance in obese Zucker rats. Am J Physiol 255: E374-E382

22. Klip A, Ramlal T, Young DA, Holloszy JO (1987) Insulin-induced translocation of glucose transporters in rat hindlimb muscles. FEBS Lett 224: $224-230$

23. Handberg A, Vaag A, Damsbo P, Beck-Nielsen H, Vinten J (1990) Expression of insulin regulatable glucose transporters in skeletal muscle from type 2 (non-insulin-dependent) diabetic patients. Diabetologia 33: 625-627

24. Handberg A, Kayser L, Høyer PE, Vinten J (1992) A substantial part of GLUT-1 in crude membranes from muscle originates from perineurial sheaths. Am J Physiol 262: E721-E727

25. Jericevic Z, Wiese B, Bryan J, Smith LC (1989) Validation of an imaging system: steps to evaluate and validate a microscope imaging system for quantitative studies. In: Taylor DL, Wang Y-L (eds) Methods in cell biology. Academic Press, New York, pp 4783 
26. Kolterman OG, Gray RS, Griffin J et al. (1981) Receptor and postreceptor defects contribute to the insulin resistance in noninsulin-dependent diabetes mellitus. J Clin Invest 68: 957-969

27. Ciaraldi TP, Kolterman OG, Scarlett JA, Kao M, Olefsky JM (1982) Role of the glucose transport system in the postreceptor defect of non-insulin dependent diabetes mellitus. Diabetes 31 : $1061-1022$

28. DeFronzo RA (1988) The triumvirate: $\beta$-cell, muscle, liver: a collusion responsible for NIDDM. Diabetes 37:667-687

29. Hirschman MF, Goodyear LJ, Wardzala LJ, Horton ED, Horton ES (1990) Identification of an intracellular pool of glucose transporters from basal and insulin-stimulated rat skeletal muscle. J Biol Chem 265: 987-991

30. Douen AG, Ramlal T, Rastogi S et al. (1990) Exercise induces recruitment of the "insulin-responsive glucose transporter". J Biol Chem 265: 13427-13430

31. Rodnick KJ, Slot JW, Studelska DR et al. (1992) Immunocytochemical and biochemical studies of GLUT 4 in rat skeletal muscle. J Biol Chem 267:6278-6285

32. Pedersen O, Bak JF, Andersen PH et al. (1990) Evidence against altered expression of GLUT 1 or GLUT 4 in skeletal muscle of patients with obesity or NIDDM. Diabetes 39:865-870

33. Kahn BB, Rosetti L, Lodish HF, Charron MJ (1991) Decreased in vivo glucose uptake but normal expression of GLUT 1 and GLUT 4 in skeletal muscle of diabetic rats. J Clin Invest 87 : 2197-2206

34. Strout HV, Vicario PP, Biswas C et al. (1990) Vanadate treatment of streptozotocin diabetic rats restores expression of the insulinresponsive glucose transporter in skeletal muscle. Endocrino$\log y 126: 2728-2732$

35. Friedman JE, Sherman WM, Reed MJ, Elton CW, Dohn GL (1990) Exercise training increases glucose transporter protein GLUT 4 in skeletal muscle of obese Zucker (fa/fa) rats. FEBS Lett 268: 13-16

36. Zarjevski N, Doyle P, Jeanrenaud B (1992) Muscle insulin resistance may not be a primary etiological factor in the genetically obese fa/fa rat. Endocrinology 130: 1564-1570
37. Koranyi L, James D, Mueckler M, Permutt MA (1990) Glucose transporter levels in spontaneously obese $(\mathrm{db} / \mathrm{db})$ insulin-resistant mice. J Clin Invest 85: 962-967

38. Slieker LJ, Sundell KL, Heath WF et al. (1992) Glucose transporter levels in tissues of spontaneously diabetic Zucker fa/fa rat (ZDF/drt) and viable yellow mouse (A $\mathrm{A}^{\mathrm{vy} / \mathrm{a}}$ ). Diabetes $41: 187-$ 193

39. Hager SR, Pastorek D, Jochen AL, Meier D (1991) Divergence between GLUT 4, RNA and protein abundance in skeletal muscle of insulin resistant rats. Biochem Biophys Res Commun 181:240-245

40. Haber RS, Weinstein SP (1992) Role of glucose transporters in glucocorticoid-induced insulin resistance. GLUT 4 isoform in rat skeletal muscle is not decreased by dexamethasone. Diabetes 41: 728-735

41. Cusin I, Terretaz J, Rohner-Jeanrenaud F, Zarjevski N, Assimacopoulos-Jeannet F, Jeanrenaud B (1990) Hyperinsulinemia increases the amount of GLUT $4 \mathrm{mRNA}$ in white adipose tissue and decreases that of muscles: a clue for increased fat depot and insulin resistance. Endocrinology 127: 3246-3248

42. Friedman JE, De Vente JE, Peterson RG, Dohm GL (1991):A.ltered expression of muscle glucose transporter GLUT 4 in diabetic fatty Zucker rats (ZDF/Drt-fa), Am J Physiol 261: E782-E788

Received: 19 November 1992

and in revised form: 11 January 1993

Dr. A. Handberg

Institute of Medical Physiology B

Panum Institute

University of Copenhagen

Blegdamsvej 3C

DK-2200 Copenhagen

Denmark 